\title{
MEDICATION ADHERENCE IN TYPE 2 DIABETIC PATIENTS: A STUDY IN SAUDI ARABIA
}

\author{
ABDULLAH K. RABBA ${ }^{1,2}$, WALEED S. ALJIRIS ${ }^{1}$, NEHAD J. AHMED ${ }^{1}$, KHALID M. ALKHARFY1,3* \\ 1Department of Clinical Pharmacy, College of Pharmacy, Prince Sattam Bin Abdulaziz University, Al-Kharj, Saudi Arabia, ${ }^{2}$ Department of \\ Pharmacy, Birzeit University, Ramallah, Palestine, ${ }^{3}$ Department of Clinical Pharmacy, King Saud University, Riyadh, Saudi Arabia \\ Email: alkharfy@ksu.edu.sa
}

Received: 06 Jan 2017 Revised and Accepted: 21 Sep 2017

\section{ABSTRACT}

Objective: To assess the extent of antidiabetic drug adherence in patients with type 2 diabetes mellitus, and different factors affecting adherence in outpatient clinics in Alkharj city, Kingdom of Saudi Arabia.

Methods: Type 2 diabetic patients attending two outpatient clinics in Alkharj city, KSA, were randomly selected and interviewed for information regarding their antidiabetic drug adherence. A six-item questionnaire was used to measure the level of adherence in study participants. Participants scoring six points are categorized as (high adherent), while scores of 4 to 5 puts the participants in the (medium adherence) category, and participants with scores less than 4 are of (Low adherence). Patient's records were also, reviewed, for relevant lab and clinical data.

Results: 68 type 2 diabetic patients participated in this study from April 2014 to September 2014. Majority of participants (73.5\%) were of 40 to 60 $\mathrm{y}$ old. Hypertension and/or lipid disorders were encountered in the majority of patients in this study.

$26.5 \%$ of patients in this study were categorized as (high adherent), $55.9 \%$ as (medium adherent), and $17.6 \%$ as (low adherent). $72 \%$ of patients had an $\mathrm{HbA1c}$ level of $\geq 7 \%$ suggesting poor control of their disease. Patients with a longer history of diabetes, and patients with a HbA1c level less than $7 \%$ found to achieve better adherence levels (p-value $<0.05$ ).

Conclusion: Antidiabetic drug adherence was successfully assessed in the sample under investigation. Subjective information from patients suggested accepted a level of adherence. Nevertheless, objective measurements of HbA1c, suggest poor glycemic control that possibly reflects poor adherence to antidiabetic medications.

Keywords: Diabetes mellitus, Adherence, Non-adherence, Alkharj city, Saudi Arabia

(C) 2017 The Authors. Published by Innovare Academic Sciences Pvt Ltd. This is an open access article under the CC BY license (http://creativecommons.org/licenses/by/4.0/) DOI: http://dx.doi.org/10.22159/ijpps.2017v9i11.16963

Diabetes mellitus (DM) is one of the most widely encountered chronic diseases in the world, and the number of adults with diabetes has more than doubled over the past three decades [1]. Several factors are known to increase the occurrence of diabetes mellitus, especially in developing countries, such as obesity, inactive life, ageing of the population, and unhealthy diet [2,3].

The prevalence of diabetes among the population of Saudi Arabia is very high with a major clinical and public health burden[2]. The prevalence of diabetes in Saudi Arabia has increased from around $10 \%$ in 1989 to one-third of the population in 2009[4]. A large proportion of diabetic patients in Saudi Arabia found to be unaware of having DM $[2,5]$

Drug adherence also referred to as drug compliance is defined as the degree or extent of conformity to the recommendations about dayto-day treatment by health care provider regarding the timing, dosage, and frequency of the medications [6].

Previous studies have demonstrated that non-compliance or nonadherence was one of the major contributing factors leading to poor glycemic control $[7,8]$.

The application of pharmaceutical care plans were shown to exhibit favourable effects on medication adherence and glycemic control of people with type $2 \mathrm{DM}$, with the main determinants of therapeutic success in the management of diabetes are adherence to diabetes medications and lifestyle modification [9].

In a retrospective cohort study in Scotland, only one-third of patients with Type 2 diabetes had adequate adherence to oral hypoglycemic medications, and once daily administration was associated with greater adherence than multiple daily administrations [10]. Causes of medication non-adherence or noncompliance can be attributed to all or one of the following: patient, treatment, or, health care provider [11].
Direct and indirect methods are used to measure therapeutic adherence. Direct methods of measuring therapeutic adherence include measuring the level of a drug or its metabolite in urine or blood [12]. We can also use the achievement of treatment goals as objective information to measure therapeutic adherence [13]. The most common and the simplest methods for measuring medication adherence are indirect methods, like patient self-reports and patient interviews [14].

Several studies assessing adherence and factors contributing to nonadherence to diabetes medications in arab countries have been published $[4,13,15-21]$. Nevertheless, only few studies on medication adherence among Saudi diabetics have been published $[4,16-18]$, none of these studies were conducted in Alkharj city in Saudi Arabia. Due to limited researchers regarding this important issue in Saudi population, this study aims to assess the rate of medication adherence, different factors affecting it, and its impact on glycemic control among type 2 DM patients in Alkharj city, Saudi Arabia.

Type 2 diabetic patients using oral anti-diabetic medications, and attending two outpatient clinics in Alkharj city, Saudi Arabia, namely, university hospital outpatient clinics at Prince Sattam bin Abdulaziz University and Seih medical centre, were interviewed for information regarding their antidiabetic drug adherence, and drug use behaviors that may affect their adherence. 68 patients, who fulfilled the above criteria and who consented to participate in this study, were randomly selected during the period from April 2014 to September 2014.

In light of previously published methods assessing medication adherence [16, 22], a six-item questionnaire was designed, validated, and used to measure the level of adherence in study participants. The six items used for assessment of adherence are as follows: do you have adequate knowledge about diabetes? Do you have good 
communication with health care provider? Do you monitor your blood glucose level regularly? Do you fill your diabetes medication on a regular basis? Have you missed your diabetes medication before? Do you usually talk about your medication problems with your healthcare provider?

Upon filling the questionnaire, patients were given a score from 0 to 6. Responders who scored six points are categorized as (high adherent), while scores of 4 to 5 puts the participants in the (medium adherence) category, and participants with scores less than 4 are of (low adherence). Patient's records were also, reviewed, for information on glycated haemoglobin A1c (HbA1c) level, and other lab results.

This study was approved by the ethical committee of college of pharmacy of Prince Sattam Bin Abdulaziz University. Consent was obtained from all patients participating in this study prior to participation.

Data were analyzed using Statistical Package for Social Sciences (SPSS) version 22. Data are presented in numbers $(\mathrm{N})$ and percentages (\%) unless otherwise mentioned. Chi-square test was used to assess the statistical significance. $P$ values less than 0.05 were considered to represent the statistically significant difference between adherence and the covariate under study.

Study sample consisted of 68 patients with type 2 diabetes. Majority of participants (73.5\%) were of 40 to $60 \mathrm{y}$ old. Hypertension and/or lipid disorders were encountered in $83 \%$ of patients in this study. Complete demographic characteristics of this study sample including, gender, age, nationality, marital status, educational level, diabetes history, and co-morbid conditions, are shown in table 1.

Table 1: Demographic characteristics of subjects given as number (percentage) $(n=68)$

\begin{tabular}{|c|c|}
\hline Patient's demographics & N (\%) \\
\hline \multicolumn{2}{|l|}{ Gender } \\
\hline Male & $39(57.4 \%)$ \\
\hline Female & $29(42.6 \%)$ \\
\hline \multicolumn{2}{|l|}{ Age } \\
\hline $30-40$ & $11(16.2 \%)$ \\
\hline $40-60$ & $50(73.5 \%)$ \\
\hline$>60$ & $7(10.3 \%)$ \\
\hline \multicolumn{2}{|l|}{ Nationality } \\
\hline Saudi & $49(72.1 \%)$ \\
\hline Non-Saudi & $19(27.9 \%)$ \\
\hline \multicolumn{2}{|l|}{ Marital status } \\
\hline Single & $5(7.40 \%)$ \\
\hline Married & $63(92.6 \%)$ \\
\hline \multicolumn{2}{|l|}{ Educational level } \\
\hline No formal education & $17(25.0 \%)$ \\
\hline School level & $33(48.5 \%)$ \\
\hline University level & $17(25.0 \%)$ \\
\hline \multicolumn{2}{|l|}{ Diabetes history } \\
\hline Less than $5 y$ & $15(22.1 \%)$ \\
\hline 5 to $10 y$ & $26(38.2 \%)$ \\
\hline More than $10 \mathrm{y}$ & $26(38.2 \%)$ \\
\hline \multicolumn{2}{|l|}{ Co-morbid conditions } \\
\hline Hypertension & $10(14.7 \%)$ \\
\hline Dyslipidemia & $25(36.8 \%)$ \\
\hline Both & $21(30.9 \%)$ \\
\hline None & $12(17.6 \%)$ \\
\hline
\end{tabular}

$n=68$

Table 2: Characteristics of medication regimes and behaviors given as number (percentage) $(n=68)$

\begin{tabular}{|c|c|}
\hline Variables & $\mathbf{N}(\%)$ \\
\hline \multicolumn{2}{|c|}{ Number of diabetes medications } \\
\hline One & $4(5.9 \%)$ \\
\hline Two & $47(69.1 \%)$ \\
\hline Three & $11(16.2 \%)$ \\
\hline More than three & $6(8.80 \%)$ \\
\hline \multicolumn{2}{|c|}{ Medication frequency } \\
\hline Once daily & $4(5.9 \%)$ \\
\hline Twice daily & $46(67.6 \%)$ \\
\hline Three times a day & $17(25 \%)$ \\
\hline Four times a day & $1(1.5 \%)$ \\
\hline \multicolumn{2}{|c|}{ Filling medications regularly } \\
\hline Yes & $55(80.9 \%)$ \\
\hline No & $13(19.1 \%)$ \\
\hline \multicolumn{2}{|l|}{ Ever missed a dose } \\
\hline Yes & $42(62.7 \%)$ \\
\hline No & $25(37.3 \%)$ \\
\hline \multicolumn{2}{|c|}{ Usually communicate with healthcare provider } \\
\hline Yes & $63(94 \%)$ \\
\hline No & $4(6 \%)$ \\
\hline
\end{tabular}

$n=68$ 
Regarding routine monitoring of blood glucose level, 61 patients $(89 \%)$ stated that they monitor blood glucose level monthly. And concerning the knowledge about diabetes 45 patients (66\%) claimed having adequate knowledge about diabetes.

Table 2 shows different characteristics related to medication regimens used for this study participants and behaviors and practices of patients toward their prescribed antidiabetic medications.

The six-item questionnaire, described in the methodology part above, was successfully used to categorize study participants in three categories according to adherence score, namely, high adherence, medium adherence, and low adherence as shown in table
3. Table 3 also presents HbA1c levels of study participants, where $72 \%$ of patients had an HbA1c level of $\geq 7 \%$ suggesting poor control of their disease [23, 24].

HbA1c level and history of diabetes (how long have been diabetic) were shown to significantly affect level of adherence as shown in table 4. For example, $44.4 \%$ of study participants who had an $\mathrm{HbA1c}$ level of less than $7 \%$ got a high adherence score, while only $15.2 \%$ of participants with $\mathrm{HbA} 1 \mathrm{c} \geq 7 \%$ had a high adherence score $(p<0.05)$. On the other hand, $42.3 \%$ of patients with more than $10 \mathrm{y}$ history of diabetes found to achieve high adherence scores, while $20 \%$ of patients with less than $5 \mathrm{y}$ history of diabetes found to have high adherence scores $(p<0.05)$.

Table 3: Adherence and HbA1c levels for study participants: number (percentage) $(n=68)$

\begin{tabular}{ll}
\hline Variables & $\mathbf{N}(\%)$ \\
Adherence level & $18(26.5 \%)$ \\
High adherence & $38(55.9 \%)$ \\
Medium adherence & $12(17.6 \%)$ \\
Low adherence & \\
HbA1c level & $46(72 \%)$ \\
$\geq 7 \%$ & $18(28 \%)$ \\
$<7 \%$ & $18 \%$ \\
\hline
\end{tabular}

$\mathrm{n}=68$

Table 4: Factors affecting adherence level (HbA1c level and how long have been diabetic) shown as count (percentage) ( $n=68)$

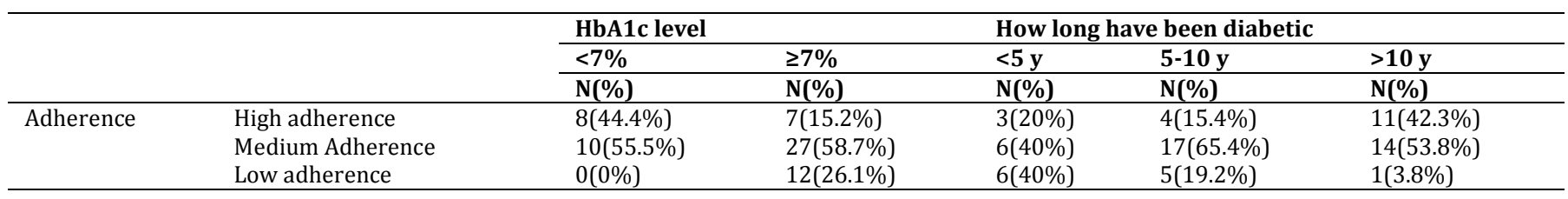

$\mathrm{n}=68, \mathrm{HbA} 1 \mathrm{c}$ mean $\% \pm \mathrm{SD} 7.6 \% \pm 1.2$

Study results revealed that different demographic characteristics are represented in the sample under investigation, i.e. males and females, those with university-level education, and those less educated, and different age groups.

The current findings showed that majority of study subjects $(82.4$ $\%)$ have other disorders in addition to diabetes, namely, hypertension, lipid disorders, or both, which agrees with previously published reports where hypertension and dyslipidaemia where shown to affect large proportion of diabetic patients in Saudi Arabia [3, 16].

Regarding adherence levels obtained for this study participants, results agree with previously published reports assessing adherence among diabetic patients in Palestine and Nigeria, where $82.4 \%$ of patients achieved moderate or high adherence level in this study, compared to $73.4 \%$ obtained by Fadare et al. [25], and $83.1 \%$ obtained by Jamous et al.[21].

While most of the study subjects said that they filled their medication regularly, $62.7 \%$ admitted that they missed medication doses before. Further, despite $26.5 \%$ and $55.9 \%$ of study participants achieved high and medium adherence levels, respectively, these data are obtained from patient's subjective information. And in order to explore the achievement of treatment goals objectively, we observed the level of HbA1c for the patients, since the achievement of treatment goals can be considered as a direct evidence for therapeutic adherence.

Patient's HbA1c levels were found to be significantly correlated with adherence levels, where patients with HbA1c levels less than $7 \%$ tend to achieve better adherence scores. In addition, duration of type $2 \mathrm{DM}$ was also shown to affect patient adherence where patients having longer history of diabetes tend to score higher adherence levels.
As mentioned, $62.7 \%$ of patient missed their medication before, in order to decrease dose missing, different strategies such as using refill reminders and advising the patients to take dosages in conjunction with routine daily activities such as before brushing of the teeth or at mealtimes, are advisable.

A small number of participants recruited in the study, is the main limitation of the study.

To conclude, antidiabetic drug adherence was successfully assessed in the sample under investigation. Adherence level of study participants to their oral antidiabetic medications, based on patient's subjective information seems to be modestly acceptable. Nevertheless, objective measurements of $\mathrm{HbA1c}$ in our study participants suggest poor glycemic control that potentially reflect poor adherence to antidiabetic medications. Thus, patient education effort is needed in Alkharj city to emphasize the importance of drug adherence in achieving treatment goals. To improve medication adherence we should assure that patients achieve continuous education about the disease and medications, applying pharmaceutical care activities, provide the patient with written and oral information, and encouraging patients to visit their health care providers regularly.

This study was approved by the ethical committee of the college of pharmacy of Prince Sattam Bin Abdulaziz University and was in accordance with the 1964 Helsinki declaration and its later amendments. Informed consent was obtained from all patients participating in this study prior to participation.

\section{ACKNOWLEDGEMENT}

Authors are grateful to health care staff of Prince Sattam Bin Abdulaziz University Hospital and Seih medical center for facilitating our work. 


\section{AUTHORS CONTRIBUTION}

Author's contribution are as follows, A Rabba: data collection, data analysis, and writing the manuscript. W. Aljiris, data collection, data analysis. N. Ahmed: data collection, data analysis. K. Alkharfy: generating the research idea, data analysis, and writing the manuscript

\section{CONFLICT OF INTERESTS}

All authors state that they have no conflict of interest to declare.

\section{REFERENCES}

1. Danaei G, Finucane MM, Lu Y, Singh GM, Cowan MJ, Paciorek CJ, et al. National, regional, and global trends in fasting plasma glucose and diabetes prevalence since 1980: systematic analysis of health examination surveys and epidemiological studies with 370 country-years and $2 \cdot 7$ million participants. Lancet 2011;378:31-40.

2. Al-Nozha MM, Al-Maatouq MA, Al-Mazrou YY, Al-Harthi SS, Arafah MR, Khalil MZ, et al. Diabetes mellitus in Saudi Arabia. Saudi Med J 2004;25:1603-10.

3. Al-Daghri NM, Al-Attas OS, Alokail MS, Alkharfy KM, Yousef M, Sabico SL, et al. Diabetes mellitus type 2 and other chronic noncommunicable diseases in the central region, Saudi Arabia (Riyadh cohort 2): a decade of an epidemic. BMC Med 2011;9:76.

4. Alharbi NS, Almutari R, Jones S, Al-Daghri N, Khunti K, de Lusignan $\mathrm{S}$. Trends in the prevalence of type 2 diabetes mellitus and obesity in the Arabian gulf states: systematic review and meta-analysis. Diabetes Res Clin Pract 2014;106:e30-3.

5. Al-Rubeaan K, Al-Manaa H, Khoja T, Ahmad N, Al-Sharqawi A, Siddiqui K, et al. The Saudi abnormal glucose metabolism and diabetes impact study (SAUDI-DM). Ann Saudi Med 2014;34:465-75.

6. Cramer JA, Roy A, Burrell A, Fairchild CJ, Fuldeore MJ, Ollendorf DA, et al. Medication compliance and persistence: terminology and definitions. Value Health 2008;11:44-7.

7. Adisa R, Alutundu MB, Fakeye TO. Factors contributing to nonadherence to oral hypoglycemic medications among ambulatory type 2 diabetes patients in Southwestern Nigeria. Pharm Pract (Granada) 2009;7:163-9.

8. Gelaw BK, Mohammed A, Tegegne GT, Defersha AD, Fromsa M, Tadesse E, et al. Nonadherence and contributing factors among ambulatory patients with antidiabetic medications in Adama Referral Hospital. J Diabetes Res 2014. http://dx.doi.org/ $10.1155 / 2014 / 617041$

9. Chung WW, Chua SS, Lai PSM, Chan SP. Effects of a pharmaceutical care model on medication adherence and glycemic control of people with type 2 diabetes. Patient Prefer Adherence 2014;8:1185-94.

10. Donnan PT, MacDonald TM, Morris AD. Adherence to prescribed oral hypoglycaemic medication in a population of patients with type 2 diabetes: a retrospective cohort study. Diabet Med 2002;19:279-84.

11. Hugtenburg JG, Timmers L, Elders PJ, Vervloet M, van Dijk L. Definitions, variants, and causes of nonadherence with medication: a challenge for tailored interventions. Patient Prefer Adherence 2013;7:675-82.

12. Osterberg L, Blaschke T. Adherence to medication. N Engl J Med 2005;353:487-97.

13. Shams MEE, Barakat EAME. Measuring the rate of therapeutic adherence among outpatients with T2DM in Egypt. Saudi Pharm J 2010;18:225-32.

14. Girerd X, Hanon O, Anagnostopoulos K, Ciupek C, Mourad JJ, Consoli S. Assessment of antihypertensive compliance using a self-administered questionnaire: development and use in a hypertension clinic. Presse Med 2000;30:1044-8.

15. Hamooz S, Sweileh W, Aker O. Effect of "Polypharmacy" and "Frequency of drug dosing" on rate of compliance among diabetic and hypertensive patients: a survey study in palestine. An-Najah Univ J Res-Nat Sci 2003;17:155-65.

16. Al-Aujan S, Al-Aqeel S, Al-Harbi A, Al-Abdulltif E. Patients' satisfaction with diabetes medications in one hospital, Saudi Arabia. Patient Prefer Adherence 2012;6:735-40.

17. Al-Hayek Aa, Robert Aa, Alzaid Aa, Nusair HM, Zbaidi NS, AlEithan $\mathrm{MH}$, et al. Association between diabetes self-care medication adherence, anxiety, depression, and glycemic control in type 2 diabetes. Saudi Med J 2012;33:681-3.

18. Khan AR, Al-Abdul Lateef ZN, Al Aithan MA, Bu-Khamseen MA, Al Ibrahim I, Khan SA. Factors contributing to non-compliance among diabetics attending primary health centers in the $\mathrm{Al}$ Hasa district of Saudi Arabia. J Family Community Med 2012;19:26-32.

19. Jarab AS, Almrayat R, Alqudah S, Thehairat E, Mukattash TL, Khdour $\mathrm{M}$, et al. Predictors of non-adherence to pharmacotherapy in patients with type 2 diabetes. Int J Clin Pharm 2014;36:725-33.

20. Jarab AS, Alqudah SG, Mukattash TL, Shattat G, Al-Qirim T. Randomized controlled trial of clinical pharmacy management of patients with type 2 diabetes in an outpatient diabetes clinic in Jordan. J Manag Care Pharm 2012;18:516-26.

21. Jamous RM, Sweileh WM, Abu-Taha AS, Sawalha AF, Zyoud SH, Morisky DE. Adherence and satisfaction with oral hypoglycemic medications: a pilot study in palestine. Int I Clin Pharm 2011;33:942-8

22. Morisky DE, Green LW, Levine DM. Concurrent and predictive validity of a self-reported measure of medication adherence. Med Care 1986;24:67-74.

23. Roquer J, Giralt-Steinhauer E, Cerdà G, Rodríguez-Campello A, Cuadrado-Godia E, Jimenez-Conde J, et al. Glycated hemoglobin value combined with initial glucose levels for evaluating mortality risk in patients with ischemic stroke. Cerebrovasc Dis $2015 ; 40: 244-50$

24. Abebe SM, Berhane Y, Worku A, Alemu S, Mesfin N. Level of sustained glycemic control and associated factors among patients with diabetes mellitus in Ethiopia: a hospital-based cross-sectional study. Diabetes Metab Syndr Obes: Targets Ther 2015;8:65-71.

25. Fadare J, Olamoyegun M, Gbadegesin BA. Medication adherence and direct treatment cost among diabetes patients attending a tertiary healthcare facility in Ogbomosho, Nigeria. Malawi Med J 2015;27:65-70. 